\title{
Sound pressure level attenuation provided by thin rigid screens coupled to tall buildings
}

\author{
A. Tadeu*, J. António, P. Amado Mendes, L. Godinho \\ Department of Civil Engineering, Faculty of Sciences and Technology, University of Coimbra, Pólo II, Rua Luís Reis Santos, \\ 3030-788 Coimbra, Portugal
}

Received 28 November 2005; received in revised form 14 February 2007; accepted 19 February 2007

Available online 1 May 2007

\begin{abstract}
This paper computes the sound pressure attenuation provided by thin rigid screens placed on the walls of a tall building to protect the building from the direct sound incidence produced by sound pressure sources placed in its vicinity. The problem is formulated in the frequency domain via the Traction Boundary Element Method (TBEM), which overcomes the thin-body difficulty arising with the classical Boundary Element Method (BEM) formulation. The building, the screens and the ground are assumed to be infinitely long and rigid. The Green's functions used in the TBEM formulation allow the solution to be obtained without discretizing the flat solid ground and vertical solid façade. Thus, only the boundary of each rigid screen is modelled, which allows the TBEM to be efficient even at high excitation frequencies. The hypersingular integrals that result from the implementation of the TBEM are computed analytically. The algorithm is verified using a BEM model, which incorporates the Green's functions for a full space, thus requiring the full discretization of the domain. The model developed is then used to simulate wave propagation in the vicinity of thin rigid screens with different dimensions and geometries. The two-dimensional (2D) and three-dimensional (3D) time and frequency responses and sound pressure attenuation results are both computed over grids of receivers placed perpendicular and parallel to the building wall.
\end{abstract}

(C) 2007 Elsevier Ltd. All rights reserved.

\section{Introduction}

Acoustic barriers are engineering solutions that block or reduce the sound pressure level in acoustic spaces. They are frequently used for tackling the problem of outside noise from traffic in the vicinity of residential and office buildings, and also to reduce the noise that workers are exposed to in confined industrial environments.

Predicting the behavior of acoustic barriers has, over the years, prompted many researchers to find ways to estimate their efficiency. Empirical methods have been proposed to solve practical engineering problems. However, given their limitations in predicting the sound pressure near acoustic screens, various numerical techniques have been developed.

\footnotetext{
*Corresponding author. Tel.: + 351239797201 ; fax: + 351239797190.

E-mail address: tadeu@dec.uc.pt (A. Tadeu).
} 
Diffraction-based methods are among the techniques that have been used to model the sound propagation near acoustic obstacles and to calculate the resulting energy loss [1-3].

Numerical methods, such as the Boundary Element Method (BEM) and the Finite Element Method, have provided more accurate results for solving the wave equation. But using these methods to solve very high frequencies involves high/prohibitive computational costs. Based on the theory of slender bodies, Filippi and Dumery [4] and Terai [5] proposed a boundary integral equation technique to model the scattering of sound waves by thin rigid screens in unbounded regions. Kawai and Terai [6] extended that method to enable the prediction of sound attenuation by rigid barriers over a totally reflective ground surface. Duhamel [7] evaluated the three-dimensional (3D) sound pressure around an acoustic barrier of constant but arbitrary cross-section erected on rigid ground. This model was further developed by Duhamel and Sergent [8] to account for absorption by the ground. Using a two-dimensional (2D) BEM, Morgan et al. [9] studied the influence of the shape and surface absorption of railway noise barriers, and compared their numerical prediction with the results given by the standard UK prediction method. A dual boundary element formulation for analyzing the 2D sound propagation in the vicinity of acoustic barriers, over an infinite plane, was proposed by Lacerda et al. [10]. Later, the 3D sound propagation around an absorptive screen was analyzed, making use of a dual boundary element formulation, which modelled the barrier as a simple surface [11]. Their technique overcame the difficulties associated with nearsingular integrals and near-degenerate equation systems. Their model used Green's functions that take into account the properties of the ground, so that absorptive ground can be modelled. Jean et al. [12] calculated the $3 \mathrm{D}$ responses of barriers to traffic noise as a combination of $2 \mathrm{D}$ solutions, considering point sources, coherent and incoherent line sources. Their model can ascribe absorbing properties to the acoustic barrier and to the ground surface.

The BEM is a suitable tool to model wave propagation in unbounded and semi-unbounded media, since it automatically satisfies the far-field radiation conditions and requires only the discretization of the material discontinuities. The authors of this paper have recently published work on the propagation of sound waves in the presence of acoustic barriers placed close to very tall buildings, using the BEM. That model uses appropriate Green's functions, derived using the image source technique, which allows the problem to be solved by discretizing only the lateral faces of the barrier and its upper part. Different geometries were analyzed, representing the cases of a single building, two buildings forming a corner ( $L$ configuration) and three buildings defining a laterally confined space ( $U$ configuration) $[13,14]$. The $L$ and $U$ configurations were solved adopting virtual sources placed along the longitudinal direction of the barrier $(z)$ in order to simulate the confinements produced by the lateral buildings. These $3 \mathrm{D}$ problems were also solved as a sequence of $2 \mathrm{D}$ problems with different spatial wavenumbers $k_{z}$, after the application of a spatial Fourier transform along the $z$ direction. That was possible because the geometry of the problem remains constant along that direction (2D geometry), while the emitting source is $3 \mathrm{D}$. This is often referred to as a two-and-a-half-dimensional problem (or 2-1/2D). Similar techniques were later adopted by Branco et al. [15] to study the behavior of differentshaped barriers, and by Tadeu and Godinho [16] in the analysis of elastic barriers.

The use of the technique described above, using the BEM, fails for very thin screens. Different approaches have been proposed over the years to overcome this difficulty. Pointer et al. [17] used an indirect boundary element formulation to analyze the seismic wavefield scattered from empty, elastic and fluid-filled fractures. The traction boundary integral equation method is another formulation derived from the fracture mechanics and wave propagation areas to overcome the thin-body difficulty [18-21]. Prosper [22] and Prosper and Kausel [23] used the Traction Boundary Element Method (TBEM) to model the 2D propagation of waves in the vicinity of $2 \mathrm{D}$ flat and horizontal empty cracks of zero thickness in elastic media. An indirect approach was proposed for the analytical evaluation of integrals with hypersingular kernels.

In the present work, the TBEM is used to compute the acoustic scattering of a sound source by thin rigid screens coupled to the walls of tall buildings, so as to provide sound attenuation in the vicinity of their windows. The rigid screens are assumed to be infinitely long and non-absorbing, and the buildings are modelled as an infinite vertical rigid barrier. The computations are performed in the frequency domain and time signatures are obtained by means of inverse Fourier transforms. All the analyses are performed using complex frequencies to avoid the aliasing phenomena. The effect of the use of complex frequencies is fully removed by rescaling the time results. 
This paper first presents the definition of the 3D problem and describes the formulation of the TBEM. Then the TBEM results are verified against those obtained by the BEM solution, when modelling regular (not thin) rigid inclusions. The computation of the time domain responses is briefly described. Finally, the model is used to compute the 2D and 3D pressure fields generated by a point pressure source in the vicinity of a tall building, for different thin rigid screen configurations. Both sound attenuation results and time responses are presented for different-sized and -shaped rigid screens, coupled to tall buildings.

\section{Problem definition}

A harmonic pressure source placed in a spatially uniform fluid medium generates a pressure field that can be expressed by

$$
p_{\text {inc }}\left(x, y, z, x_{s}, y_{s}, \omega\right)=\frac{A \mathrm{e}^{\mathrm{i}(\omega / \alpha)\left(\alpha t-\sqrt{\left(x-x_{s}\right)^{2}+\left(y-y_{s}\right)^{2}+z^{2}}\right)}}{\sqrt{\left(x-x_{s}\right)^{2}+\left(y-y_{s}\right)^{2}+z^{2}}}
$$

in which $\omega$ is the angular frequency, $\left(x_{s}, y_{s}, 0\right)$ is the position of the source, the subscript inc represents the incident field, $A$ is the wave amplitude, $\alpha$ is the pressure wave velocity of the medium, and $\mathrm{i}=\sqrt{-1}$.

Performing a Fourier transformation of Eq. (1), in the $z$ direction, and defining the effective wavenumbers

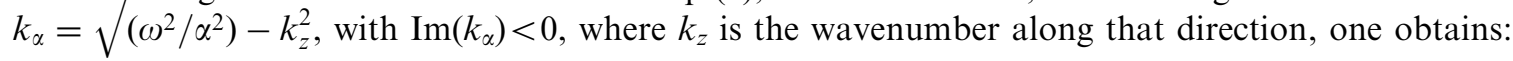

$$
\hat{p}_{\text {inc }}\left(x, y, x_{s}, y_{s}, k_{z}, \omega\right)=\frac{-\mathrm{i} A}{2} H_{0}^{(2)}\left(k_{\alpha} \sqrt{\left(x-x_{s}\right)^{2}+\left(y-y_{s}\right)^{2}}\right)
$$

in which $H_{n}^{(2)}(\cdots)$ are second Hankel functions of order $n$.

Assuming the presence of an infinite set of equally spaced sources along the $z$ direction, the above incident field can be written as

$$
p_{\text {inc }}\left(x, y, z, x_{s}, y_{s}, \omega\right)=\frac{2 \pi}{L_{v s}} \sum_{m=-\infty}^{\infty} \hat{p}_{\text {inc }}\left(x, y, x_{s}, y_{s}, k_{z}, \omega\right) \mathrm{e}^{-\mathrm{i} k_{z} z},
$$

where $L_{v s}$ is the spatial source interval, and $k_{z}=\left(2 \pi / L_{v s}\right) m$. The 3D pressure field can therefore be computed as the pressure irradiated by a sum of harmonic (steady-state) line sources, whose amplitude varies sinusoidally in the third dimension.

In the present work, the source is placed in an acoustic medium bounded by two perpendicular flat surfaces, one simulating the horizontal ground surface, and the other representing the façade of a tall building. To avoid the discretization of these two orthogonal interfaces, the pressure field defined by Eq. (3) needs to be rewritten in a way that satisfies null normal velocities at those boundaries. This can be accomplished by adding

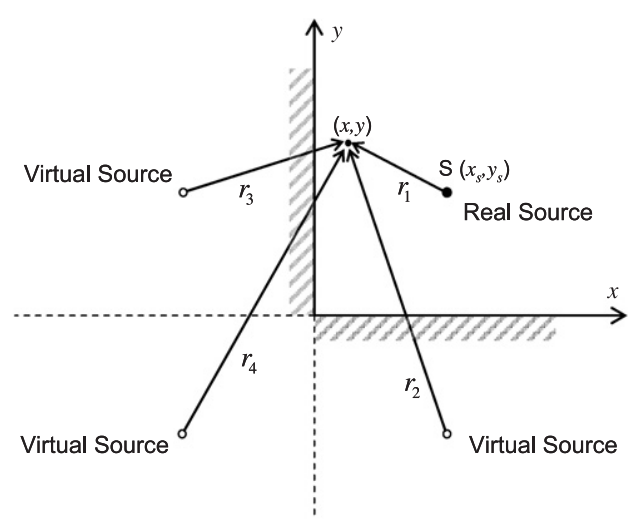

Fig. 1. Illustration of the image source technique. 
the pressure field generated by the real source to that produced by three virtual sources (image sources), located in such a way that they act as mirrors in relation to the vertical and horizontal planes (see Fig. 1). When the vertical plane and the horizontal plane are placed at $x=0$ and $y=0$, respectively, the final incident pressure field is given by

$$
\hat{p}_{\text {inc }}\left(x, y, x_{s}, y_{s}, k_{z}, \omega\right)=\sum_{j=1}^{4} \frac{-\mathrm{i} A}{2}\left[H_{0}^{(2)}\left(k_{\alpha} r_{j}\right)\right]
$$

in which

$$
\begin{aligned}
& r_{1}=\sqrt{\left(x-x_{s}\right)^{2}+\left(y-y_{s}\right)^{2}}, \\
& r_{2}=\sqrt{\left(x-x_{s}\right)^{2}+\left(y+y_{s}\right)^{2}}, \\
& r_{3}=\sqrt{\left(x+x_{s}\right)^{2}+\left(y-y_{s}\right)^{2}}, \\
& r_{4}=\sqrt{\left(x+x_{s}\right)^{2}+\left(y+y_{s}\right)^{2}} .
\end{aligned}
$$

In the acoustic domain defined above, thin rigid screens are coupled to the façade of a tall building to produce sound attenuation in the vicinity of its windows.

\section{Traction boundary element formulation}

Consider the case of a fluid inclusion bounded by a surface $S$, submerged in a homogeneous fluid medium, defined by $x \geqslant 0 \wedge y \geqslant 0$, which is excited by the incident pressure field given by $p_{\text {inc }}$, placed outside the inclusion. The classical boundary integral equation, written along the boundary through the exterior domain, can be derived from the Helmholtz equation in the frequency domain by applying the reciprocity theorem, leading to

$$
\begin{aligned}
c p\left(x_{0}, y_{0}, k_{z}, \omega\right)= & \int_{S} q\left(x, y, \mathbf{n}_{n}, k_{z}, \omega\right) G\left(x, y, x_{0}, y_{0}, k_{z}, \omega\right) \mathrm{ds} \\
& -\int_{S} H\left(x, y, \mathbf{n}_{n}, x_{0}, y_{0}, k_{z}, \omega\right) p\left(x, y, k_{z}, \omega\right) \mathrm{d} s+\hat{p}_{\text {inc }}\left(x_{0}, y_{0}, x_{s}, y_{s}, k_{z}, \omega\right),
\end{aligned}
$$

where $G$ and $H$ represent the Green's functions for the pressure $(p)$ and pressure gradient $(q)$, at a point $(x, y)$ on the boundary $S$ due to a virtual point pressure source at a collocation point $\left(x_{0}, y_{0}\right)$. $\mathbf{n}_{n}$ represents the unit outward normal along the boundary $S$ and the factor $c$ assumes the value $1 / 2$ if $\left(x_{0}, y_{0}\right) \in S$ and $S$ is smooth

$$
\hat{p}_{\text {inc }}\left(x_{0}, y_{0}, x_{s}, y_{s}, k_{z}, \omega\right)=\sum_{j=1}^{4} \frac{-\mathrm{i} A}{2}\left[H_{0}^{(2)}\left(k_{\alpha} r_{s j}\right)\right]
$$

corresponds to the pressure incident field at $\left(x_{0}, y_{0}\right)$, when the point pressure source is located at $\left(x_{s}, y_{s}\right)$, with

$$
\begin{aligned}
& r_{s 1}=\sqrt{\left(x_{0}-x_{s}\right)^{2}+\left(y_{0}-y_{s}\right)^{2}}, \\
& r_{s 2}=\sqrt{\left(x_{0}-x_{s}\right)^{2}+\left(y_{0}+y_{s}\right)^{2}}, \\
& r_{s 3}=\sqrt{\left(x_{0}+x_{s}\right)^{2}+\left(y_{0}-y_{s}\right)^{2}}, \\
& r_{s 4}=\sqrt{\left(x_{0}+x_{s}\right)^{2}+\left(y_{0}+y_{s}\right)^{2}} .
\end{aligned}
$$


For 2-1/2D problems in an acoustic medium defined by $x \geqslant 0 \wedge y \geqslant 0$, the Green's functions for pressure and pressure gradients in Cartesian coordinates can be given by

$$
\begin{aligned}
& G\left(x, y, x_{0}, y_{0}, k_{z}, \omega\right)=\frac{\mathrm{i}}{4} \sum_{j=1}^{4}\left[H_{0}^{(2)}\left(k_{\alpha} r_{0 j}\right)\right], \\
& H\left(x, y, \mathbf{n}_{n}, x_{0}, y_{0}, k_{z}, \omega\right)=\frac{-\mathrm{i}}{4} \sum_{j=1}^{4}\left[k_{\alpha} H_{1}^{(2)}\left(k_{\alpha} r_{0 j}\right) \frac{\partial r_{0 j}}{\partial \mathbf{n}_{n}}\right]
\end{aligned}
$$

with

$$
\begin{aligned}
& r_{01}=\sqrt{\left(x_{0}-x\right)^{2}+\left(y_{0}-y\right)^{2}}, \\
& r_{02}=\sqrt{\left(x_{0}-x\right)^{2}+\left(y_{0}+y\right)^{2}}, \\
& r_{03}=\sqrt{\left(x_{0}+x\right)^{2}+\left(y_{0}-y\right)^{2}}, \\
& r_{04}=\sqrt{\left(x_{0}+x\right)^{2}+\left(y_{0}+y\right)^{2}} .
\end{aligned}
$$

Notice that the use of these Green's functions avoids the discretization of the vertical rigid plane $(x=0)$ and the horizontal rigid plane $(y=0)$. When we consider the case of a rigid inclusion, null normal pressure gradients are imposed along the boundary $S$, simplifying the boundary integral Eq. (5) to

$$
c p\left(x_{0}, y_{0}, k_{z}, \omega\right)=-\int_{S} H\left(x, y, \mathbf{n}_{n}, x_{0}, y_{0}, k_{z}, \omega\right) p\left(x, y, k_{z}, \omega\right) \mathrm{d} s+\hat{p}_{\mathrm{inc}}\left(x_{0}, y_{0}, x_{s}, y_{s}, k_{z}, \omega\right) .
$$

However, a standard BEM formulation cannot be used to calculate the $3 \mathrm{D}$ scattering field generated by very thin rigid screens, since it degenerates. A TBEM formulation is therefore proposed to overcome that difficulty.

The traction boundary integral equation can be derived by applying the gradient operator to the boundary integral Eq. (8), (which can be seen as assuming the existence of dipole pressure sources):

$$
\begin{aligned}
\operatorname{ap}\left(x_{0}, y_{0}, k_{z}, \omega\right)= & -\int_{S} \bar{H}\left(x, y, \mathbf{n}_{n}, x_{0}, y_{0}, \mathbf{n}_{0 j}, k_{z}, \omega\right) p\left(x, y, k_{z}, \omega\right) \mathrm{d} s \\
& +\bar{p}_{\text {inc }}\left(x_{0}, y_{0}, \mathbf{n}_{01}, x_{s}, y_{s}, k_{z}, \omega\right) .
\end{aligned}
$$

In this equation, $\bar{H}\left(x, y, \mathbf{n}_{n}, x_{0}, y_{0}, \mathbf{n}_{0 j}, k_{z}, \omega\right)$ are the Green's functions for pressure gradients generated by dipole pressure sources. These may be seen as resulting from the derivatives of the pressure gradients for monopole sources, at point $(x, y)$, caused by a virtual concentrated pressure source acting at the collocation point $\left(x_{0}, y_{0}\right)$. The factor $a$ is null for piecewise straight boundary elements, as noted by Guiggiani [24].

The solutions of these equations are defined by discretizing the boundary into $N$ straight boundary elements, with one nodal point in the middle of each element. In order to avoid the discretization of the vertical plane $(x=0)$ and the horizontal plane $(y=0)$, the required $2-1 / 2 \mathrm{D}$ Green's functions for pressure gradients are defined as

$$
\begin{aligned}
\bar{H}\left(x, y, \mathbf{n}_{n}, x_{0}, y_{0}, \mathbf{n}_{0 j}, k_{z}, \omega\right) \\
=\frac{\mathrm{i}}{4} k_{\alpha} \sum_{j=1}^{4}\left\{\left\{-k_{\alpha} H_{2}^{(2)}\left(k_{\alpha} r_{0 j}\right)\left[\left(\frac{\partial r_{0 j}}{\partial x}\right)^{2} \frac{\partial x}{\partial \mathbf{n}_{n}}+\frac{\partial r_{0 j}}{\partial x} \frac{\partial r_{0 j}}{\partial y} \frac{\partial y}{\partial \mathbf{n}_{n}}\right]\right.\right. \\
\left.\left.+\frac{H_{1}^{(2)}\left(k_{\alpha} r_{0 j}\right)}{r_{0 j}}\left[\frac{\partial x}{\partial \mathbf{n}_{n}}\right]\right\} \frac{\partial x}{\partial \mathbf{n}_{0 j}}\right\}+\frac{\mathrm{i}}{4} k_{\alpha} \sum_{j=1}^{4}\left\{\left\{-k_{\alpha} H_{2}^{(2)}\left(k_{\alpha} r_{0 j}\right)\left[\frac{\partial r_{0 j}}{\partial x} \frac{\partial r_{0 j}}{\partial y} \frac{\partial x}{\partial \mathbf{n}_{n}}\right.\right.\right. \\
\left.\left.\left.+\left(\frac{\partial r_{0 j}}{\partial y}\right)^{2} \frac{\partial y}{\partial \mathbf{n}_{n}}\right]+\frac{H_{1}^{(2)}\left(k_{\alpha} r_{0 j}\right)}{r_{0 j}}\left[\frac{\partial y}{\partial \mathbf{n}_{n}}\right]\right\} \frac{\partial y}{\partial \mathbf{n}_{0 j}}\right\}
\end{aligned}
$$




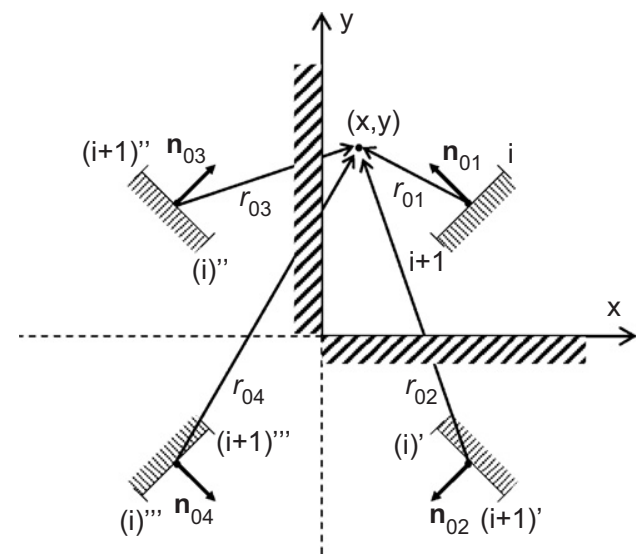

Fig. 2. Definition of $r_{0 j}$ and $\mathbf{n}_{0 j}$.

where $r_{0 j}$ and $\mathbf{n}_{n}$ are defined as above, while $\mathbf{n}_{0 j}$ is the unit outward normal of the real boundary at $\left(x_{0}, y_{0}\right)$, $(j=1)$, and of the fictitious boundaries and collocation points obtained by mirroring the real ones in relation to the vertical $(x=0)$ and horizontal $(y=0)$ planes (see Fig. 2).

The incident field $\bar{p}_{\text {inc }}\left(x_{0}, y_{0}, \mathbf{n}_{01}, x_{s}, y_{s}, k_{z}, \omega\right)$, when the source is located at $\left(x_{s}, y_{s}\right)$, can be computed as

$$
\bar{p}_{\text {inc }}\left(x_{0}, y_{0}, \mathbf{n}_{01}, x_{s}, y_{s}, k_{z}, \omega\right)=\frac{\mathrm{i} A}{2} \sum_{j=1}^{4}\left[k_{\alpha} H_{1}^{(2)}\left(k_{\alpha} r_{s j}\right)\left(\frac{\partial r_{s j}}{\partial x} \frac{\partial x}{\partial \mathbf{n}_{01}}+\frac{\partial r_{s j}}{\partial y} \frac{\partial y}{\partial \mathbf{n}_{01}}\right)\right] .
$$

The integrations in the boundary integral equation are performed analytically when the element being integrated is the loaded one and $j=1$. Then, the following integral resulting from Eqs. (9) and (10) becomes hypersingular

$$
\begin{aligned}
\int_{C}\left\{\bar{H}\left(x, y, \mathbf{n}_{n}, x_{0}, y_{0}, \mathbf{n}_{0 j}, k_{z}, \omega\right)\right\}_{j=1} \mathrm{~d} C \\
=\int_{C} \frac{\mathrm{i}}{4} k_{\alpha}\left[-k_{\alpha} H_{2}^{(2)}\left(k_{\alpha} r_{01}\right)\left(\frac{\partial r_{01}}{\partial x} \frac{\partial x}{\partial \mathbf{n}_{n}}+\frac{\partial r_{01}}{\partial y} \frac{\partial y}{\partial \mathbf{n}_{n}}\right)^{2}+\frac{H_{1}^{(2)}\left(k_{\alpha} r_{01}\right)}{r_{01}}\right] \mathrm{d} C .
\end{aligned}
$$

This integral can be evaluated analytically, considering the dynamic equilibrium of a semi-cylinder bounded by the boundary element, leading to

$$
\begin{aligned}
\int_{C}\{ & \left.\bar{H}\left(x, y, \mathbf{n}_{n}, x_{0}, y_{0}, \mathbf{n}_{0 j}, k_{z}, \omega\right)\right\}_{j=1} \mathrm{~d} C \\
= & \int_{0}^{\pi} \frac{\mathrm{i}}{4} k_{\alpha}\left[-\frac{H_{1}^{(2)}\left(k_{\alpha} r_{01}\right)}{r_{01}}+k_{\alpha} H_{0}^{(2)}\left(k_{\alpha} r_{01}\right)\right] \frac{L}{2} \sin \theta \mathrm{d} \theta \\
& +\rho \omega^{2} \frac{\mathrm{i}}{4} k_{\alpha} \int_{0}^{L / 2} H_{1}^{(2)}\left(k_{\alpha} r_{01}\right) r_{01} \mathrm{~d} r_{01} \int_{0}^{\pi} \sin \theta \mathrm{d} \theta \\
= & \frac{\mathrm{i}}{2}\left(k_{\alpha}\right)^{2}\left[\int_{0}^{L / 2} H_{0}^{(2)}\left(k_{\alpha} r_{01}\right) \mathrm{d} r_{01}-\frac{1}{k_{\alpha}} H_{1}^{(2)}\left(k_{\alpha} \frac{L}{2}\right)\right],
\end{aligned}
$$

where $L$ stands for the length of the boundary element $C$ and $\rho$ represents the mass density of the fluid medium. The integral $\int_{0}^{L / 2} H_{0}^{(2)}\left(k_{\alpha} r_{01}\right) \mathrm{d} r_{01}$ is evaluated as

$$
\int_{0}^{L / 2} H_{0}^{(2)}\left(k_{\alpha} r_{01}\right) \mathrm{d} r_{01}=\frac{L}{2} H_{0}^{(2)}\left(k_{\alpha} \frac{L}{2}\right)+\pi \frac{L}{4}\left[H_{1}^{(2)}\left(k_{\alpha} \frac{L}{2}\right) S_{0}\left(k_{\alpha} \frac{L}{2}\right)-H_{0}^{(2)}\left(k_{\alpha} \frac{L}{2}\right) S_{1}\left(k_{\alpha} \frac{L}{2}\right)\right],
$$

where $S_{n}(\cdots)$ are Struve functions of order $n$. 
All other integrations in the boundary integral equation are computed through a Gaussian quadrature scheme.

The procedure described above leads to a system of equations that relates the pressure field to a set of nodal pressure values. The nodal pressure values are calculated after this system of equations has been solved. The scattered pressure field at any point inside the acoustic medium can then be calculated in relation to the nodal pressure values calculated previously.

\section{Verification of the TBEM algorithm}

The TBEM algorithm is verified by taking a cylindrical circular rigid inclusion, placed inside an acoustic domain bounded by vertical and horizontal planes located at $x=0.0 \mathrm{~m}$ and $y=0.0 \mathrm{~m}$, respectively. These planes are assumed to be rigid and filled at $x>0 \wedge y>0$ with an unbounded homogeneous acoustic medium $(\alpha=340 \mathrm{~m} / \mathrm{s})$. The axis of the cylinder is placed at $(3.0,1.5 \mathrm{~m})$, and has a radius of $1.0 \mathrm{~m}$. This system, represented in Fig. 3(a), is excited by two harmonic cylindrical pressure sources (with $k_{z}=0 \mathrm{rad} / \mathrm{m}$ and $\left.k_{z}=12 \mathrm{rad} / \mathrm{m}\right)$ at point $\mathrm{O}(1.0,1.0 \mathrm{~m})$.

The responses are recorded at a receiver placed at $(1.0,5.0 \mathrm{~m})$, for frequencies ranging from 5 to $1280 \mathrm{~Hz}$. These responses are compared with those provided by a BEM code that uses Green's functions for an unbounded medium. In this case, the geometry of the problem is built up using the real rigid cylinder and pressure source, and its mirror images in relation to the vertical and horizontal planes, $x=0.0 \mathrm{~m}$ and $y=0.0 \mathrm{~m}$ (see Fig. 3(b)).

Fig. 4 displays the real and imaginary parts of the scattered complex pressure at the receiver for the BEM and TBEM models, when 250 constant boundary elements were used to model each inclusion and the system was excited by each of the pressure sources. In the $2 \mathrm{D}$ situation $\left(k_{z}=0 \mathrm{rad} / \mathrm{m}\right)$, results in terms of the insertion loss (IL) values (in dB), comparing the case with a cylindrical inclusion and the case without, are presented in Fig. 5. Analyses of the results show a very good agreement between the numerical solutions obtained by the two models.

(a)

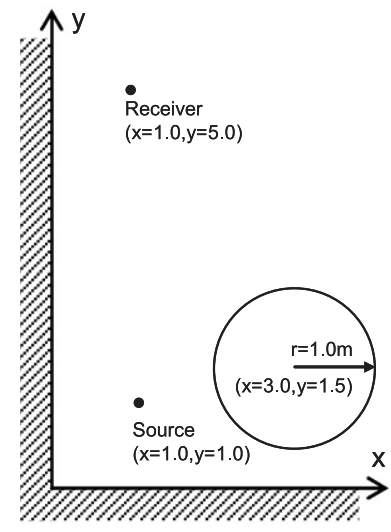

(b)

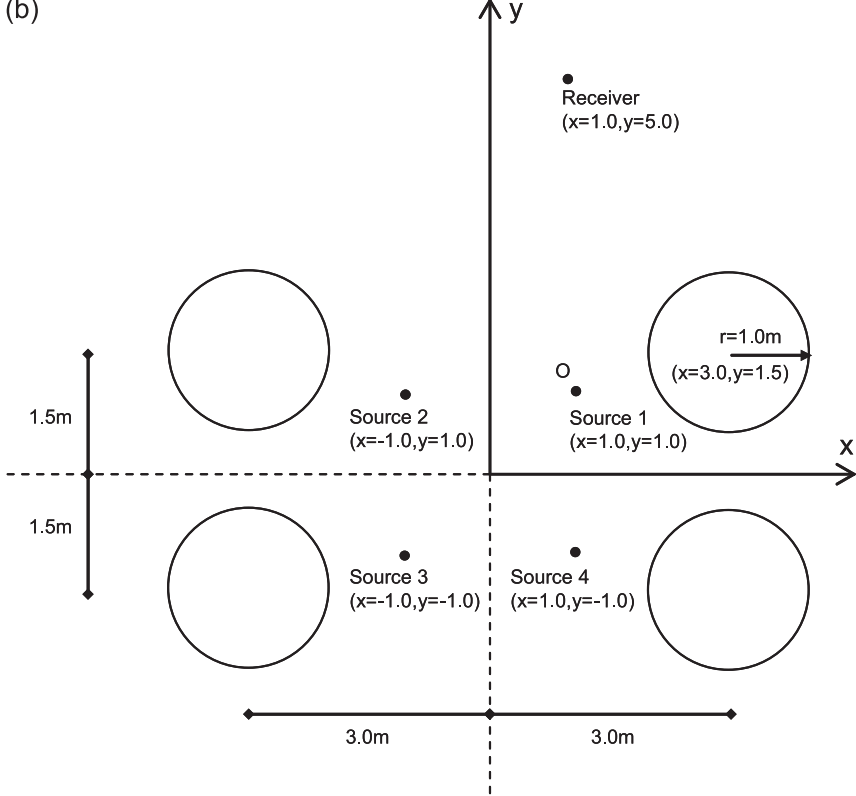

Fig. 3. Verification models: (a) geometry used in the TBEM model; (b) geometry used in the BEM model. 

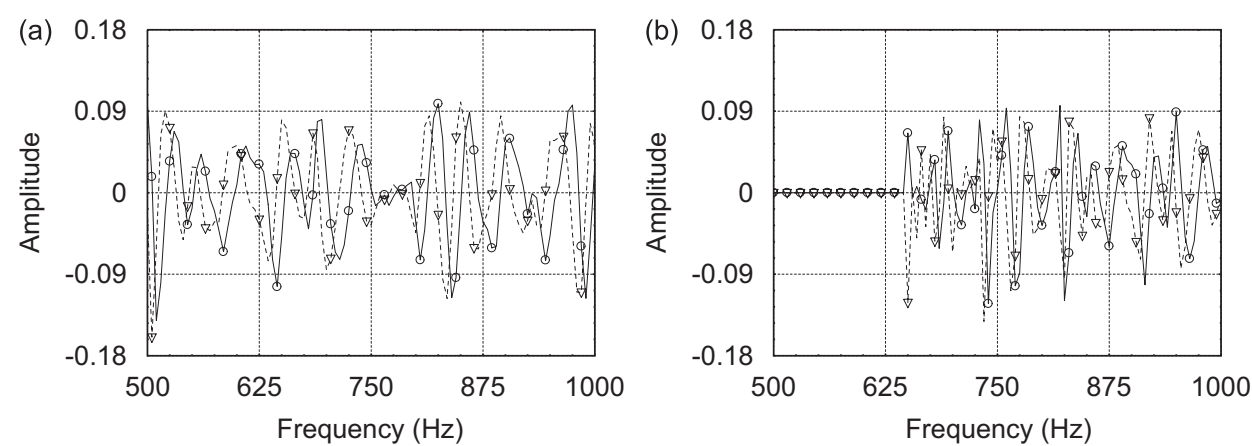

Fig. 4. TBEM verification: scattered complex pressure results computed by BEM and TBEM models, when the systems are excited by $2-1 /$ 2D pressure sources with: (a) $k_{z}=0 \mathrm{rad} / \mathrm{m}$; (b) $k_{z}=12 \mathrm{rad} / \mathrm{m}\left(\left(--_{-}\right)\right.$BEM-imaginary part, ( $\left.\longrightarrow\right)$ BEM-real part, ( $\left.\nabla\right)$ TBEMimaginary part and $(O)$ TBEM-real part).
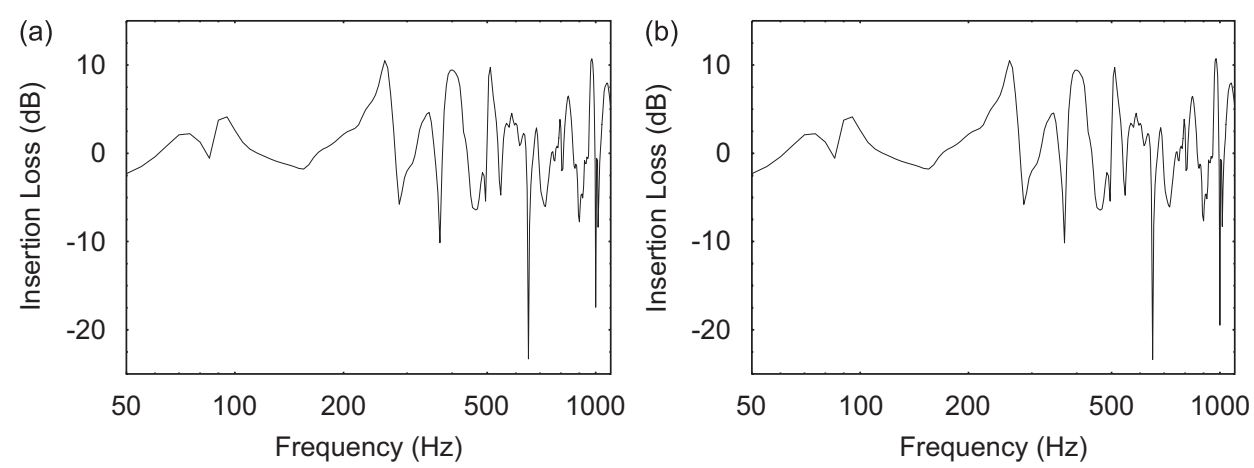

Fig. 5. TBEM validation in terms of insertion loss values (case with cylindrical inclusion compared with case without) provided by BEM and TBEM models, when the system is excited by a 2D pressure source $\left(k_{z}=0 \mathrm{rad} / \mathrm{m}\right)$ : (a) TBEM model; (b) BEM model.

\section{Pressure in time domain}

The analysis of the signals in the time domain is useful for interpreting the wave propagation phenomena in the acoustic medium, from the emitting source to the building façade, in the presence and in the absence of the acoustic screens. After computing the results in the frequency domain for different $k_{z}$ wavenumbers, the response for a point harmonic source is found by applying a discrete Fourier transformation in the wavenumber domain. This is equivalent to adding sources at spatial intervals of $L_{v s}=2 \pi / \Delta k_{z}$ (where $\Delta k_{z}$ represents the wavenumber increment).

Next, time responses are obtained using inverse fast Fourier transformations in $\omega$. A Ricker pulse is modelled since it decays rapidly in both the time and frequency domains, allowing a useful reduction in computational effort and an easier interpretation of the time signatures. The Ricker function is given by

$$
u(\tau)=A\left(1-2 \tau^{2}\right) \mathrm{e}^{-\tau^{2}}
$$

in which $A$ is the amplitude, $\tau=\left(t-t_{s}\right) / t_{0}$ and $t$ represent the time, with $t_{s}$ being the time when the maximum occurs, while $\pi t_{0}$ is the dominant wavelet period.

To prevent the contamination of the response by the periodic sources (aliasing phenomena), the spacing between them must be large enough to ensure that their contribution arrives at times later than $T=2 \pi / \Delta \omega$ (where $\Delta \omega$ is the frequency increment). This is achieved by using complex frequencies with an imaginary part of the form $\omega_{c}=\omega-\mathrm{i} \eta$. For the imaginary part of the angular frequency, $\eta=0.7 \Delta \omega$ was chosen to attenuate the wraparound by a factor of $\mathrm{e}^{0.7 \Delta \omega T}=81$. This value of $\eta$ is commonly used in wave propagation analysis. 
The use of a larger value would introduce loss of accuracy in the response, and it should not be much smaller because the aim is to achieve a maximum reduction in the contribution of the aliasing phenomena. This procedure also leads to the minimization or even the elimination of the virtual sources' contribution. The effect of the use of complex frequencies is taken into account by rescaling the response in the time domain, applying an exponential window $\mathrm{e}^{\eta t}[25,26]$.

\section{Numerical examples}

The procedure described in the above sections was used to study the acoustic behavior of rigid acoustic screens, attached to a tall building over a rigid floor. A set of seven thin rigid screens, attached to the façade, separated from one another by a $3.0 \mathrm{~m}$ gap, and with the lowest one placed $4.0 \mathrm{~m}$ above the floor (see Fig. 6(a)), is modelled using single lines of boundary elements. Three acoustic screen geometries were studied: horizontal rectilinear screens, inclined rectilinear screens and curvilinear screens, as illustrated in Figs. 6(b)-(d). For all the geometries, the horizontal dimension of the screen is kept constant at $2.0 \mathrm{~m}$.

The host acoustic medium is air, exhibiting a density of $1.22 \mathrm{~kg} / \mathrm{m}^{3}$ and allowing a pressure wave velocity of $340.0 \mathrm{~m} / \mathrm{s}$. A pressure source, placed $0.6 \mathrm{~m}$ above the ground, $10.0 \mathrm{~m}$ away from the building façade and at $z=0.0 \mathrm{~m}$, generates acoustic waves that excite the system. The responses were computed over a 3D grid of $101 \times 101 \times 101$ receivers, regularly distributed throughout the propagation domain, at intervals of $0.25,0.25$ and $0.375 \mathrm{~m}$ along the $x, y$ and $z$ directions, respectively.

The time domain responses allow both the interpretation of the scattering produced by the rigid acoustic screens, and analysis of the attenuation provided by the acoustic screens. To obtain time solutions, the computations were performed in the frequency range of $[2.5,2560.0 \mathrm{~Hz}]$. A frequency step of $2.5 \mathrm{~Hz}$ was used, defining a maximum time domain window of $0.4 \mathrm{~s}$. For the calculation of time domain responses, the source was assumed to generate a Ricker pulse, with dominant periods corresponding to characteristic frequencies of either 250,500 or $1000 \mathrm{~Hz}$. These results are presented as a set of snapshots taken over the planes of the receiver grids, using a grayscale ranging from black to white as the amplitude changes from negative to positive values.

The sound isolation provided by the rigid screens attached to the building façade was determined by evaluating the peak sound pressure level $\left(L_{p k}\right)$ [27] attenuation over the different receiver grid planes defined previously. This $L_{p k}$ attenuation can be seen as the difference between the $L_{p k}$ registered with and without the presence of the screens. The relevant peak sound pressure levels are calculated taking into account the maximum instantaneous absolute pressure value registered at each receiver in the time responses, and applying

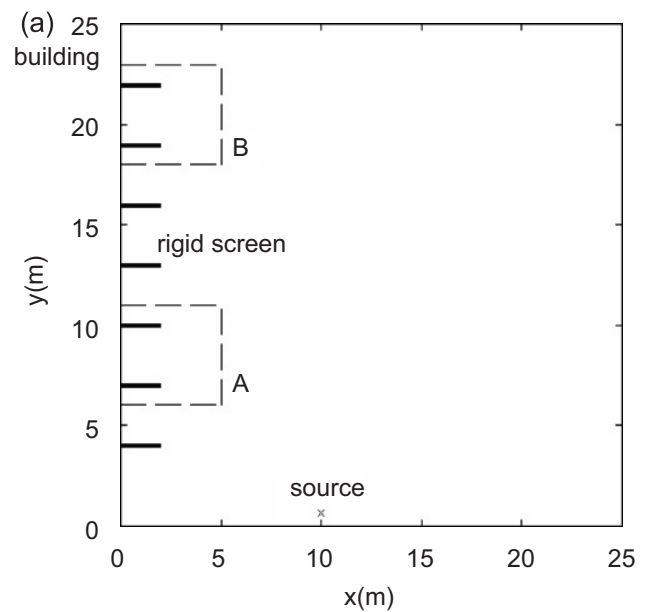

(b) (c)

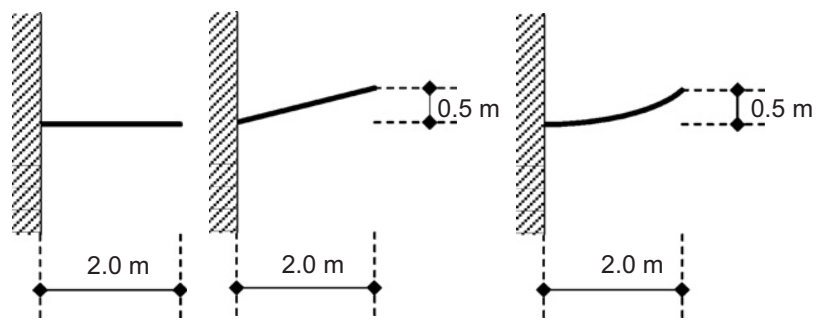

Fig. 6. (a) Global geometry of the propagation medium, with A and B representing zooms taken near the building façade. Geometric definition of the three kinds of rigid screens attached to the building: (b) horizontal rectilinear screen; (c) inclined rectilinear screen; (d) circumference-arc-shaped screen. 
the expression $L_{p k}=10 \log \left[\left|p_{\max }\right|^{2} /\left(2 \times 10^{-5} \mathrm{~Pa}\right)^{2}\right]$. In the results presented here, characteristic frequencies of 250,500 and $1000 \mathrm{~Hz}$ have been adopted.

Each screen is modelled using an appropriate number of elements defined by the relation between the wavelength and the length of the boundary elements, which was set at 10 . The number of elements used for each screen is never less than 20.

The rest of this section is divided into two parts. In the first, 2D analyses are performed in a vertical plane perpendicular to the building façade to evaluate the performance of the differently shaped screens. This made it possible to select the geometry to be used for the 3D results.

\subsection{Two-dimensional analyses}

\subsubsection{IL spectra at specific receivers}

Frequency domain results from the TBEM model computations are used to exemplify the outcome at specific receiver positions near the building façade. The case of horizontal rectilinear screens attached to the façade is adopted to present these results in terms of IL values (comparing the cases with and without the rigid screens) in the range of the computed frequencies [2.5, 2560.0 Hz]. Fig. 7 exhibits the IL at receivers located near the façade $(x=0.25 \mathrm{~m})$, at three different heights $y=8.5,14.5$ and $20.5 \mathrm{~m}$. In these plots, negative values correspond to attenuation caused by the presence of the screens. The acoustic field spectrum is very irregular along the frequency range with a set of peaks and dips at several frequencies due to the interaction of the different pulses with the rigid façade and screens. In the same figure, the IL curve in $1 / 3$ octave bands is also presented for bands with central frequencies from 50 to $2000 \mathrm{~Hz}$. As expected, these curves have a smoother behavior along the frequency domain, since they correspond to the integration of the narrow band responses in wider $1 / 3$ octave bands. The interpretation of these curves indicates that, for the presented cases, the rigid screens exhibit better acoustic performance at lower frequencies, with a peak performance value noted around a frequency of $100 \mathrm{~Hz}$. This peak is caused by destructive interference between waves that reach the corresponding receiver following different paths, and arrive at that point with phase opposition. It is also clear from these plots that the performance of the screens, in terms of IL, tends to improve for the receivers located at higher positions. This effect was, in fact, expected, revealing a more pronounced shadow effect generated by the presence of the screens when these are placed further above the ground.

\subsubsection{Time domain scattering analysis}

The horizontal rectilinear screen model is used to illustrate the results obtained in the time domain. Fig. 8 presents first the snapshots taken over one of the planes perpendicular to the building façade. At time $t=0.0 \mathrm{~ms}$, the line source (with $k_{z}=0.0 \mathrm{rad} / \mathrm{m}$ ) emits a Ricker pulse with a characteristic frequency of $250 \mathrm{~Hz}$, which propagates away from it. This pulse then hits the rigid floor, where it is reflected back to the propagating domain, forming a second pattern which closely follows the direct incident wavefront. These pulses then hit the rigid façade, where they are once again totally reflected back. At time $t=35.0 \mathrm{~ms}$, this wavefield pattern can be clearly identified, for the case without rigid acoustic screens, in Fig. 8(a). Here, the time evolution of the pressure field resulting from the interaction of the direct incident pulses and their reflections from both the ground surface and the tall building can be observed in Figs. 8(c) and (e), for later time instants. As expected, as the pressure waves propagate along the domain, their amplitude decreases as they get farther away from the source position.

When the horizontal rectilinear rigid acoustic screens are attached to the building façade, the wavefield pattern becomes much more complicated. As the wavefront reaches the screens it suffers both reflection and diffraction, leading to additional pulses noticeable in Figs. 8(b), (d), and (f). At time $t=35.0 \mathrm{~ms}$, significant differences can already be identified in the regions below the third screen. Just above the second screen, a diffracted wave can be seen travelling around the screen. Below this screen, a first reflection coming from the façade reveals a fall in the pulse amplitude, compared with Fig. 8(a). As time evolves, pronounced changes can be identified in the wavefront, corresponding to a first set of reflections from the façade. In particular, the upper part of these pulses becomes more diffuse, as a result of the presence of the multiple rigid screens. These screens are also responsible for a set of additional reflections, travelling downwards, clearly visible in Figs. 8(d) 

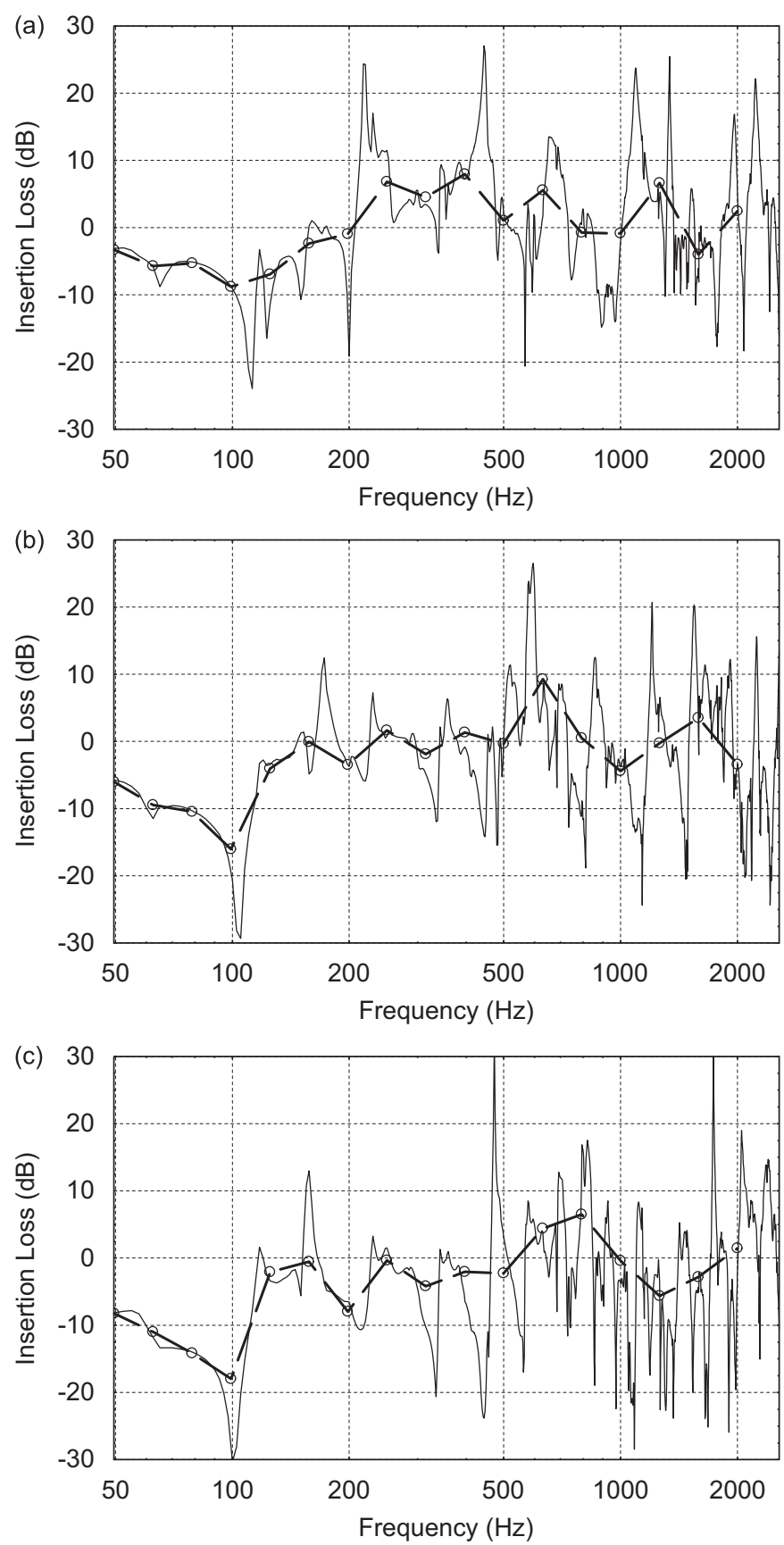

Fig. 7. Insertion loss spectra near the façade of a tall building with horizontal rectilinear rigid screens, when a line source excites the

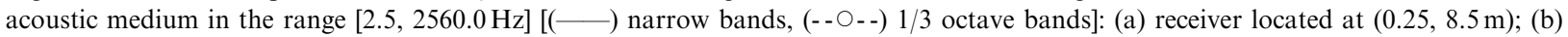
receiver located at $(0.25,14.5 \mathrm{~m})$; (c) receiver located at $(0.25,20.5 \mathrm{~m})$. See Fig. 6 (a) for building façade geometry.

and (f). In the last snapshot (Fig. 8(f)), these effects are more evident, while pulses trapped between rigid screens are visible in the response, particularly at receivers placed further from the ground.

The results obtained for the other screen geometries and for different characteristic frequencies of the source are not illustrated, since the main features of the responses were the same. 

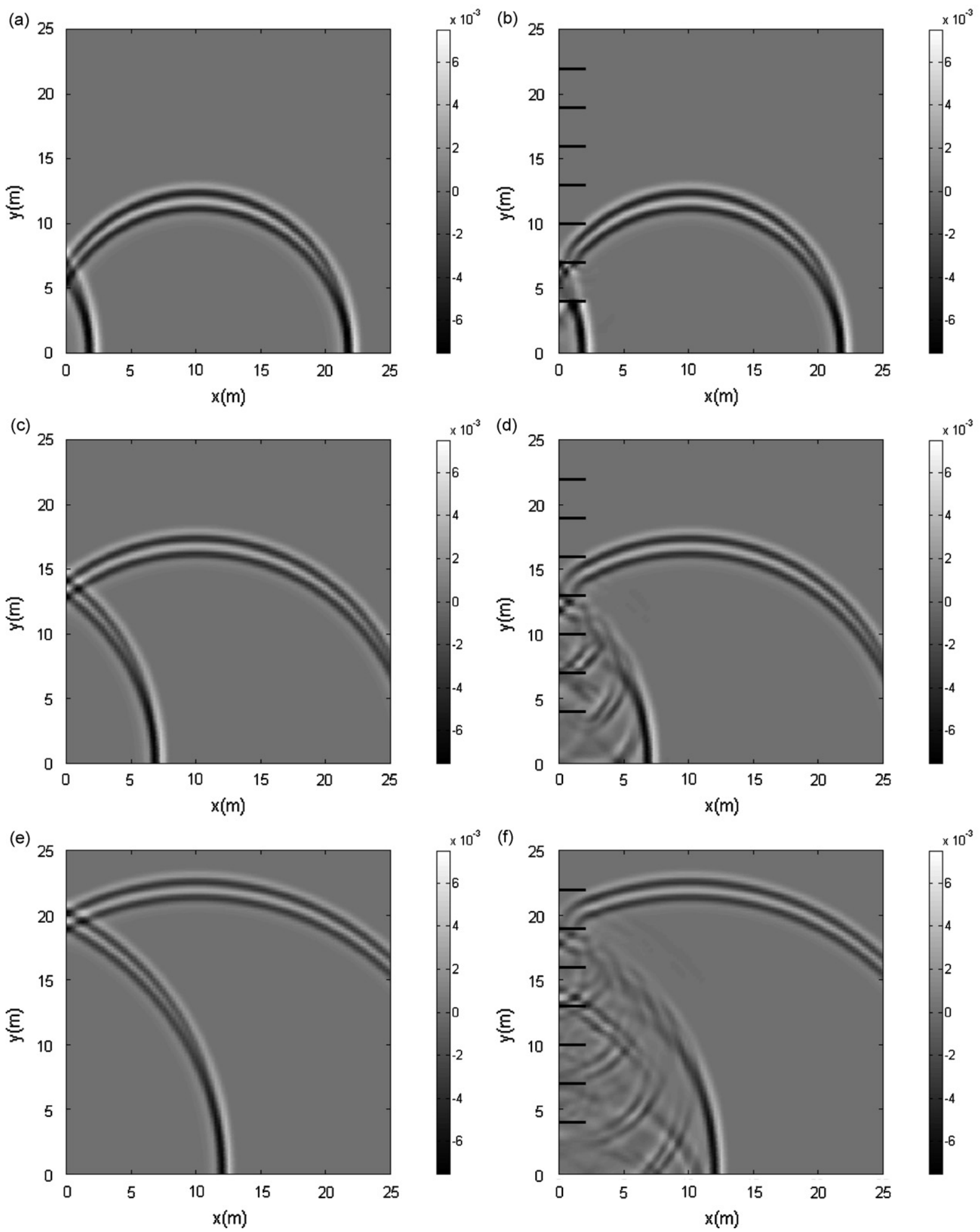

Fig. 8. Pressure wavefield evolution near a tall building, when a line source with a characteristic frequency of $250 \mathrm{~Hz}$ excites the acoustic medium: (a), (c), (e) in the absence of the rigid screens, at time instants $t=35.0,50.0$ and $65.0 \mathrm{~ms}$; (b), (d), (f) in the presence of horizontal rectilinear screens, at time instants $t=35.0,50.0$ and $65.0 \mathrm{~ms}$. See Fig. 6(a) for building façade geometry. 

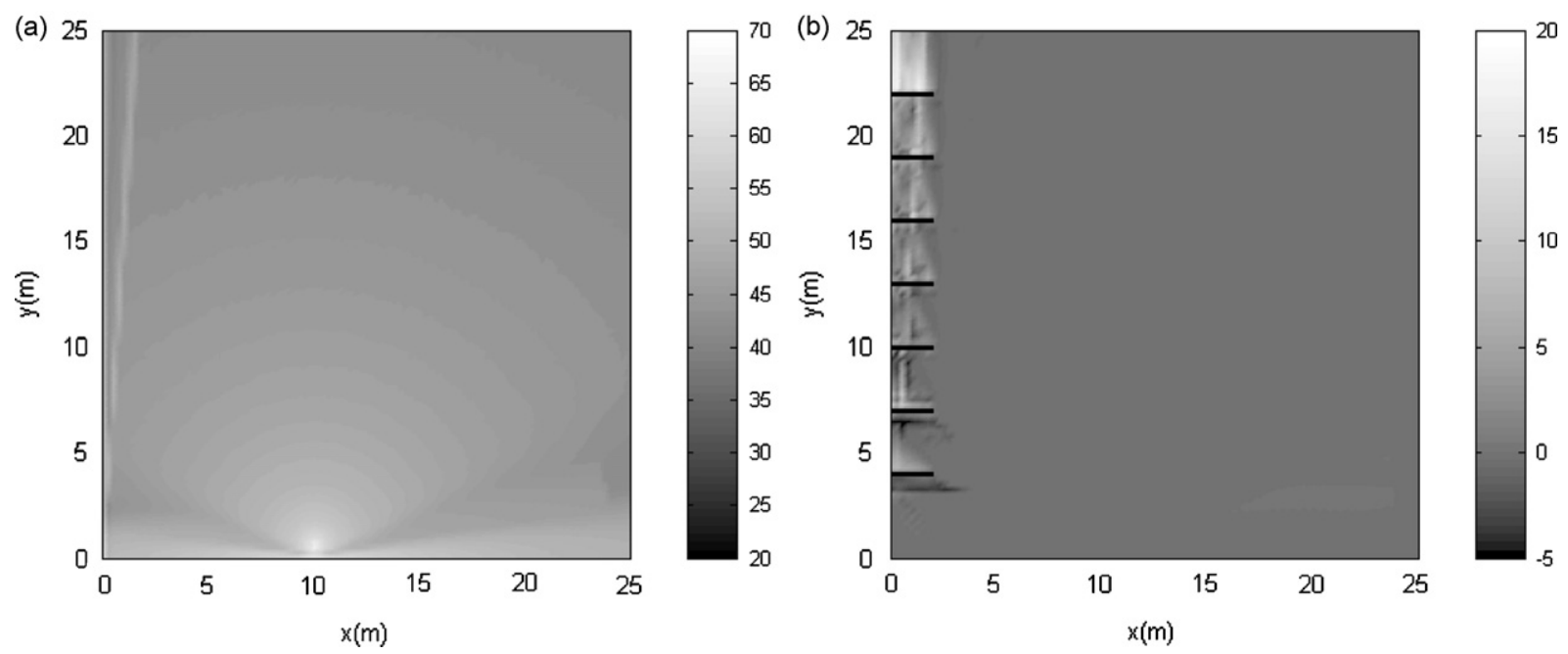

Fig. 9. Computations over the grid of receivers, for a characteristic frequency of $500 \mathrm{~Hz}$ : (a) peak sound pressure level (in dB), in the absence of the rigid screens; (b) insertion loss based on $L_{p k}$ (in $\mathrm{dB}$ ), in the presence of horizontal rectilinear screens. See Fig. 6(a) for building façade geometry.

\subsubsection{Attenuation provided by screens with different geometries}

Fig. 9(a) illustrates the $L_{p k}$ registered in the absence of the screens, for the same grids of receivers used before. As expected, the maximum values of this parameter are registered at receivers placed closer to the source, with a smooth fall being seen as receivers further away from it are considered. Since the surfaces of the building façade and the ground are totally reflective, an increase in the $L_{p k}$ can be observed at receivers very close to the façade and the ground. Additionally, a line of increased $L_{p k}$ can be seen near the façade of the building. This increase can be explained by the constructive interference occurring between pulses travelling directly from the source and pulses that suffer a first reflection at the rigid surface of the building wall.

When rigid screens are attached to the façade, there is a global fall in the $L_{p k}$ registered near the façade. Fig. 9(b) illustrates the IL based on $L_{p k}$ computed for the grid of receivers, when the horizontal rectilinear screens are modelled. Lighter shaded zones, corresponding to regions where the $L_{p k}$ decreases, are visible above the screens, particularly above the ones placed further above the ground. However, this pattern is not uniform within the area delimited by two consecutive screens, since part of the propagating energy is trapped by the rigid surfaces of the screens and the façade, giving rise to positive and negative interference between multiple pulses reflected at those surfaces. It can also be seen that, for receivers placed further above the ground, the $L_{p k}$ attenuation tends to increase. A possible explanation for this phenomenon is related to the fact that the inclination of the incident wavefront with respect to the screens tends to decrease, with a consequent increase of the sound isolation provided by the screens. A maximum attenuation is registered above the highest placed screen. This increased attenuation is explained by the behavior just described and by the fact that there are no physical barriers above this screen, so the incident pulses can propagate to the unbounded medium without the possibility of any energy becoming trapped above this screen.

Below the lowest screens, darker zones are also visible. These zones result from increased $L_{p k}$ generated by a constructive combination of directly incident pulses and pulses reflected at the lower surface of the screens.

In order to asses the influence of the shape of the screens, different simulations were carried out for the screen geometries represented in Figs. 6(b)-(d). Specific regions near the façade define two additional grids of $101 \times 101$ receivers which are identified in Fig. 6(a) as zooms A and B. The $L_{p k}$ attenuations, computed for zoom A and zoom B, are illustrated in Figs. 10(a)-(c) and Figs. 10(d)-(f), respectively. For all cases, patterns of darker and lighter shades are visible, due to the interference between different pulses travelling in this region. These patterns tend to be parallel to the rigid surfaces of the screens and façade, indicating that there is a strong interaction between waves reflected at the upper and lower screens and at the façade. A vertical line of increased attenuation can also be observed in all figures. This line indicates that the previously identified 


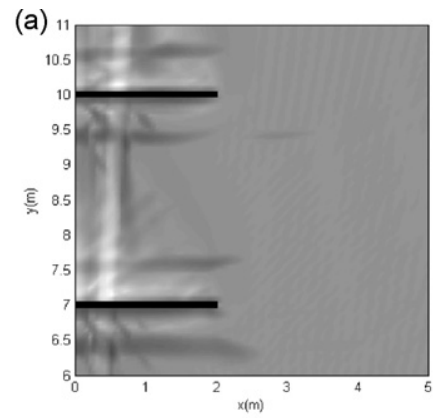

(d)

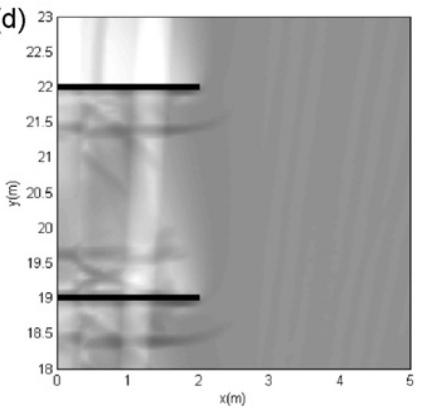

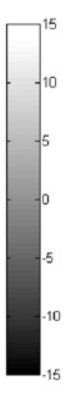

(b)
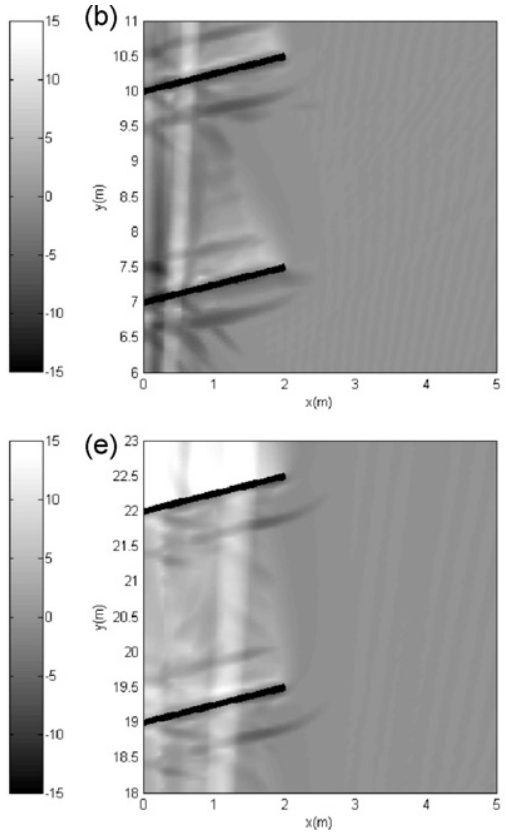
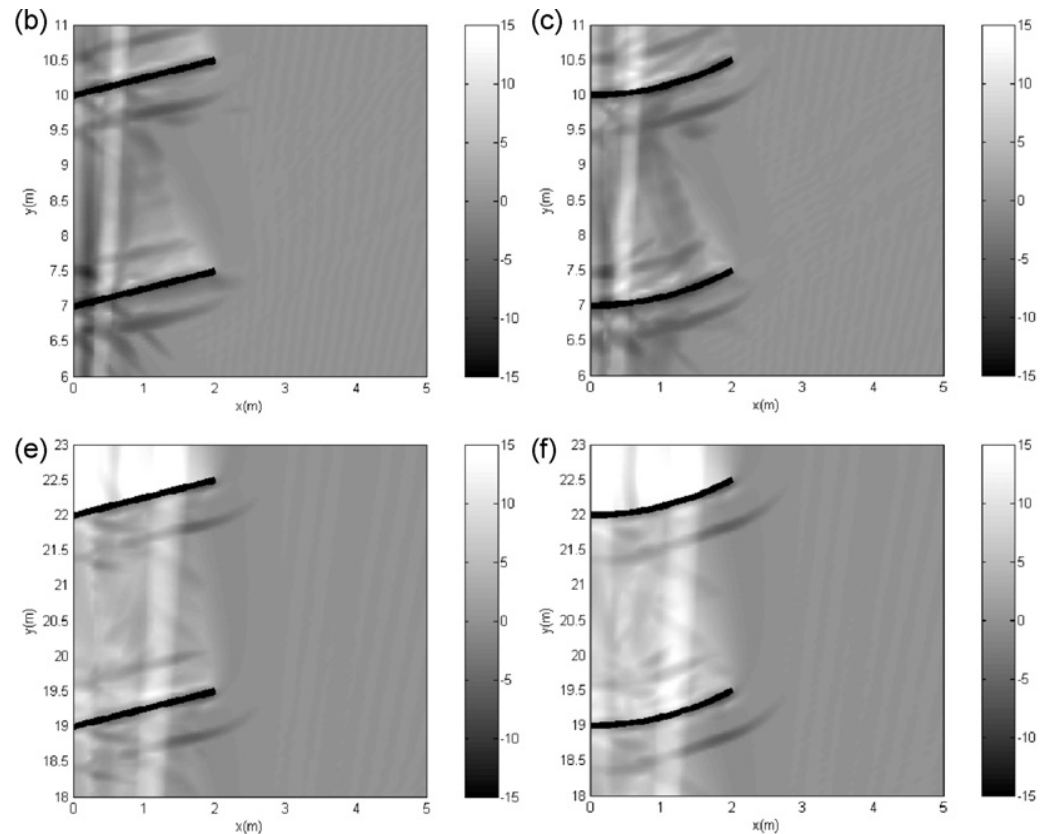

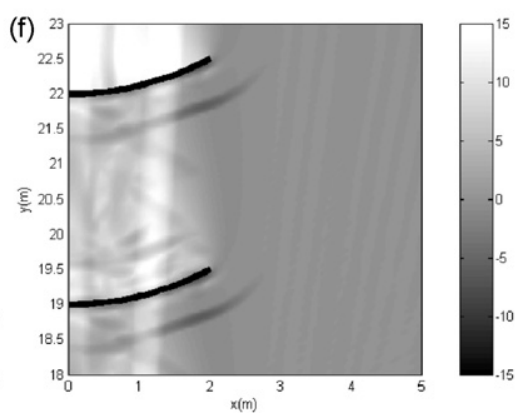

Fig. 10. Insertion loss based on $L_{p k}$ (in dB), for a characteristic source frequency of $500 \mathrm{~Hz}$, in the presence of differently shaped screens: (a)-(c) grids identified with zoom A; (d)-(f) grids identified with zoom B. See Fig. 6(a) for building façade geometry.

Table 1

Insertion loss based on spatially averaged peak SPL $\left(\bar{L}_{\mathrm{pk}}\right)$ of the differently shaped screens for characteristic frequencies of 250,500 and $1000 \mathrm{~Hz}$

Horizontal rectilinear screens $(\mathrm{dB}) \quad$ Inclined rectilinear screens $(\mathrm{dB})$

Circumference-arc-shaped screens (dB)

\begin{tabular}{crrr}
\hline$f_{c}=250 \mathrm{~Hz}$ & & & \\
Zoom A & -0.1 & -0.6 & 0.0 \\
Zoom B & 2.7 & 3.5 & 5.0 \\
$f_{c}=500 \mathrm{~Hz}$ & & & \\
Zoom A & -0.3 & -0.4 & 0.5 \\
Zoom B & 2.4 & 4.1 & 6.1 \\
$f_{c}=1000 \mathrm{~Hz}$ & & & \\
Zoom A & -0.2 & -0.8 & 0.7 \\
Zoom B & 1.4 & 3.9 & 7.0
\end{tabular}

phenomenon of constructive combination between the incident pulse and the pulse first reflected at the façade no longer occurs. Looking at the results for zoom A, only slight differences can be seen between the attenuation provided by the differently shaped screens. However, near the façade, the curvilinear screens seem to perform slightly better than the others. In the grids of receivers corresponding to the zoom B regions, the attenuation provided by the three types of screens exhibits more pronounced differences. Here again the curvilinear screens seem to perform better than the other geometries. Comparing the results obtained for zooms A and B, it is clear that at receivers placed further from the floor, the screens provide a more effective acoustic protection for the building façade.

To obtain a quantitative measure of the sound isolation provided by the screens, a numerical parameter was calculated as the difference between the spatially averaged peak sound pressure level (SPL) at receivers closer to the façade, without and with the screens (attenuation due to the presence of the screens is represented by positive values). To compute this parameter, only the responses obtained for one region, located between two 
consecutive screens, are taken into account, and the corresponding spatially averaged peak SPL $\left(\bar{L}_{p k}\right)$ is calculated as $\bar{L}_{p k}=10 \log \left[(1 / N) \sum_{n=1}^{N}\left|p_{\text {max }, n}\right|^{2} /\left(2 \times 10^{-5} \mathrm{~Pa}\right)^{2}\right]$, where $N$ is the number of receivers inside this region. This rectangular region is located in the immediate vicinity of the building, beginning $0.5 \mathrm{~m}$ above the lower screen and ending $0.5 \mathrm{~m}$ below the upper one, and has a width of $1.0 \mathrm{~m}$. For characteristic frequencies of 250,500 and $1000 \mathrm{~Hz}$, the values computed for the IL based on $\bar{L}_{p k}$ at the receiver grids identified as zoom A and zoom B are displayed in Table 1. Analysis of the computed IL values shows that, for both regions, the curvilinear screens outperform the other configurations. It can also be seen that there is a clear tendency for the IL to increase from zoom A to zoom B, corroborating the explanations given above. A similar behavior is observed for other characteristic frequencies, such as 250 and $1000 \mathrm{~Hz}$, for which only the computed IL based on $\bar{L}_{p k}$ is presented in Table 1 .

\subsection{Three-dimensional analyses}

\subsubsection{Time domain scattering analysis}

Since the curvilinear screens provided better $L_{p k}$ attenuation closer to the building façade, the pressure field excited by a point pressure source is computed and presented in Fig. 11. This figure displays the time evolution snapshots of numerical simulations at three grids of receivers ( $x=0.0 \mathrm{~m}$ (the tall building façade), $y=0.0 \mathrm{~m}$ (the ground) and $z=37.5 \mathrm{~m}$ ) when the characteristic frequency of the pulse is $250 \mathrm{~Hz}$.

In Fig. 11, the 3D behavior of the total pressure wavefield generated in the vicinity of the building is clearly perceived as time evolves after the source starts emitting at time $t=0.0 \mathrm{~ms}$. As the pulse propagates in the fluid medium away from the source point, the wave energy spreads out. The spherical incident pulses first hit the rigid floor and are totally reflected back. These pulses then hit the building façade and are again reflected back. This wavefield pattern is shown along the grids of receivers for time instants $t=35.0,89.8$ and $119.9 \mathrm{~ms}$, in the absence of the rigid screens, in Figs. 11(a), (c) and (e). The amplitude of those pressure waves falls when they propagate in the $3 \mathrm{D}$ domain, as they move further from the source point.

As before, the scattered wavefield pattern is visibly modified when the circumference-arc-shaped thin acoustic screens are attached to the tall building façade (see Figs. 11(b), (d) and (f)). The pressure wavefield plotted at the grids of receivers exhibits significant changes, due to the reflection and diffraction phenomena when the incident pulses reach the screens. These differences are slightly visible at $t=35.0 \mathrm{~ms}$, but are more pronounced for the later time instants presented. For instance, along the building façade, the wavefront presents a reduced amplitude and is no longer continuous. Smaller amplitude pulses, trapped in additional reflections by the building and the rigid screens, lead to a disturbed wave pressure field (along the $z$ direction) compared with the case of the plain building façade. This can be seen in planes corresponding to $x=0.0 \mathrm{~m}$ and $y=0.0 \mathrm{~m}$, in Figs. 11(d) and (f).

Similar behavior was also observed when numerical simulations were obtained for different characteristic frequencies of the acoustic source (not given here).

\subsubsection{Attenuation provided by screens in the building façade}

Fig. 12 illustrates the $L_{p k}$ when there are no screens, and the $L_{p k}$ attenuation provided when circumferencearc-shaped screens are added to the building façade $x=0.0 \mathrm{~m}$, for a characteristic source frequency of $250 \mathrm{~Hz}$. As before, in the absence of the rigid screens, the pressure field results from the direct incident waves interacting with the building façade and the floor surface, which are totally reflective. Therefore, the total wave pressure field corresponds to the sum of acoustic pulses, with different phases, resulting in a spatially variable peak sound pressure level, visible in Fig. 12(a) as a pattern of varying $L_{p k}$ value zones. The maximum $L_{p k}$ values are observed at receivers located nearer to the source, with a general fall in this parameter noted as the distance between the receivers and the source increases. However, a lower $L_{p k}$ zone is visible along the building façade, due to the destructive combination of the different reflected and incident pulses.

When the circumference-arc-shaped screens are attached to the building façade, the resulting IL based on $L_{p k}$ along the façade is presented in Fig. 12(b). From this figure, it is possible to conclude that a general fall in $L_{p k}$ is achieved by coupling these screens to the façade. As expected, receivers further from the floor exhibit a more pronounced $L_{p k}$ attenuation, while receivers closer to the ground give negative attenuation values, thus 

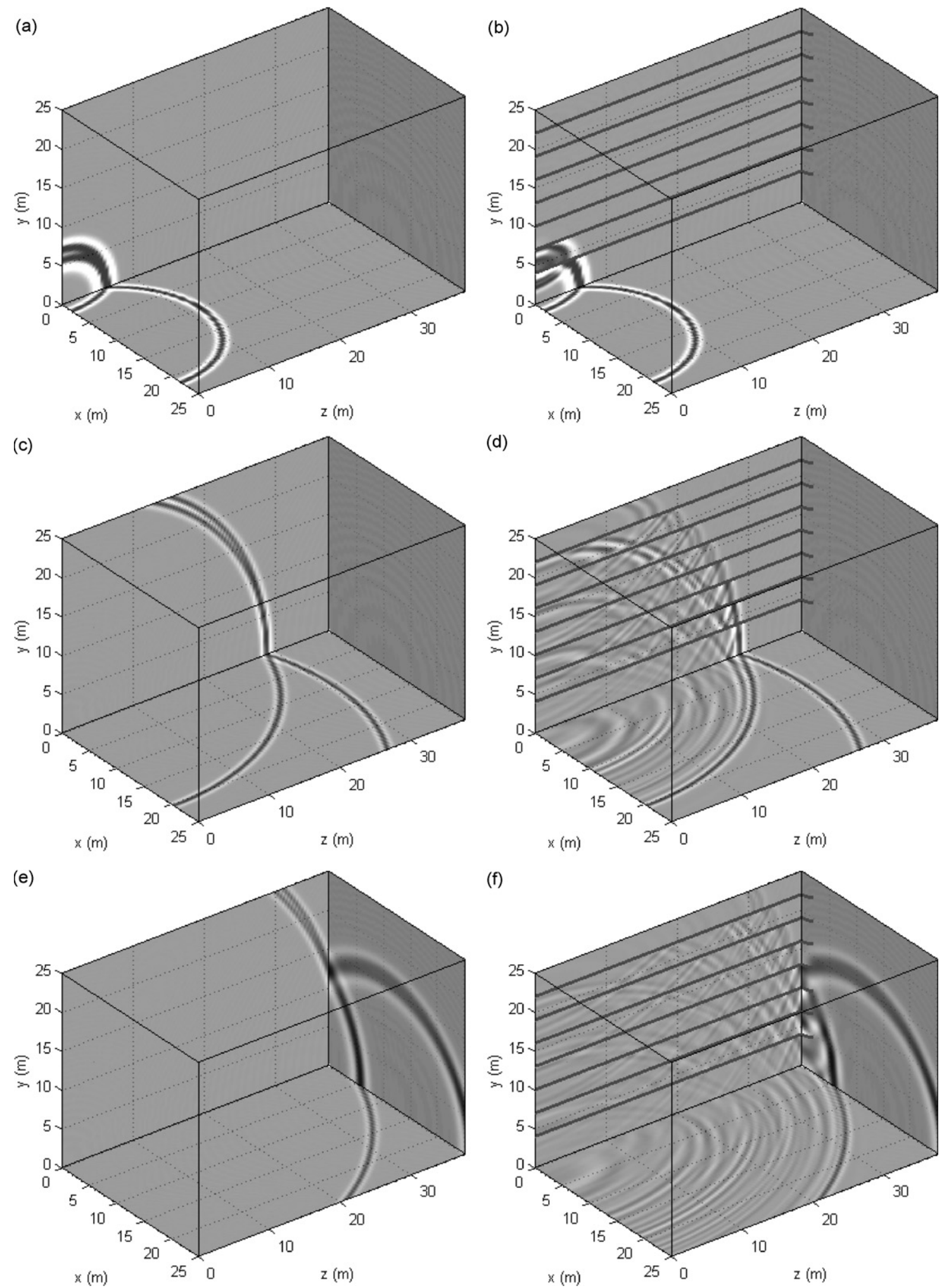

Fig. 11. Three-dimensional pressure wavefield evolution near a tall building, when a spherical source with a characteristic frequency of $250 \mathrm{~Hz}$ excites the acoustic medium: (a), (c), (e) in the absence of the rigid screens, at time instants $t=35.0,89.8$ and $119.9 \mathrm{~ms}$; (b), (d), (f) in the presence of circumference-arc-shaped screens, at the same time instants. See Fig. 6(a) for building façade geometry. 

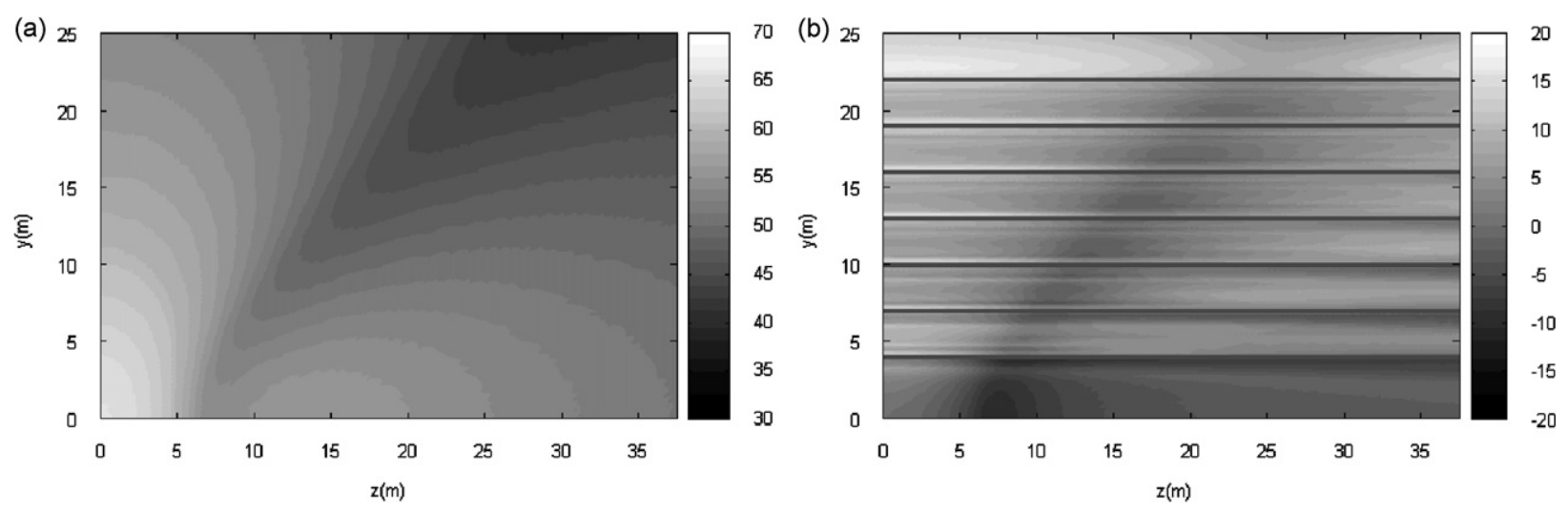

Fig. 12. Computations for the grid of receivers along the building façade, for a characteristic frequency of $250 \mathrm{~Hz}$ : (a) peak sound pressure level (in dB) without the rigid screens; (b) insertion loss based on $L_{p k}$ (in dB) with circumference-arc-shaped screens. See Fig. 6(a) for building façade geometry.

revealing that the screens have the effect of increasing the $L_{p k}$ values in this region. It is interesting to note that the poorest performance is obtained at some distance from the source plane, along the $z$ direction, in a region previously identified as a lower $L_{p k}$ zone in the absence of the screens. With this configuration, the destructive interference between incident and reflected pulses is no longer the dominant phenomenon contributing to the response, and the effect of the pulses remaining trapped below the lower placed screens seems to be determinant to the final attenuation registered at these receivers.

\section{Conclusions}

A frequency domain TBEM formulation has been presented and applied to the study of the behavior of rigid acoustic screens attached to a tall building and excited by a pressure source. The proposed model makes use of Green's functions that take into account the presence of the tall building façade and of a rigid floor, therefore making their discretization unnecessary. The model was verified against a direct BEM formulation, and was found to be stable and accurate.

A number of simulations were performed to illustrate its application to a specific case where seven rigid screens are attached to the building façade. Results for the time domain allowed the identification of the different pulses travelling in the system, and showed that the presence of the screens produces pronounced changes in the response.

Attenuation analysis was also carried out, to assess the influence of the screens' geometry and distance from the floor on the peak sound pressure levels registered near the façade. Three different configurations were tested, these being a horizontal rectilinear, an inclined rectilinear and a curvilinear geometry. The last was found to be the most effective in the acoustic protection of the façade. It was also found that the attenuation tends to increase higher up the façade. A general fall in peak sound pressure levels is observed in the façade plane along the $z$ direction when the circumference-arc-shaped screens are attached to the building. However, this decrease is not uniform along that direction. In addition, the peak sound pressure levels were found to rise in the vicinity of the lower placed screens, thus revealing their poorer performance.

\section{Acknowledgments}

This research was supported by the Portuguese Foundation for Science and Technology (FCT), under the research project POCI/ECM/58940/2004. 


\section{References}

[1] Y.W. Lam, Using Maekawa's chart to calculate finite length barrier insertion loss, Applied Acoustics 42 (1994) $29-40$.

[2] A. Muradali, K.R. Fyfe, A study of 2D and 3D barrier insertion loss using improved diffraction-based methods, Applied Acoustics 53 (1998) 49-75.

[3] L.L. Beranek, I.L. Vér, Noise and Vibration Control Engineering, Wiley Interscience, New York, 1992.

[4] P. Filippi, G. Dumery, Etude théorique et numérique de la diffraction par un écran mince, Acustica 21 (1969) $343-359$.

[5] T. Terai, On calculation of sound fields around three-dimensional objects by integral equation methods, Journal of Sound and Vibration 69 (1980) 71-100.

[6] Y. Kawai, T. Terai, The application of integral equation methods to the calculation of sound attenuation by barriers, Applied Acoustics 31 (1990) 101-117.

[7] D. Duhamel, Efficient calculation of the three-dimensional sound pressure field around a noise barrier, Journal of Sound and Vibration 197 (1996) 547-571.

[8] D. Duhamel, P. Sergent, Sound propagation over noise barriers with absorbing ground, Journal of Sound and Vibration 218 (1998) 799-823.

[9] P.A. Morgan, D.C. Hothersall, S.N. Chandler-Wilde, Influence of shape and absorbing surface-a numerical study of railway barriers, Journal of Sound and Vibration 217 (1998) 405-417.

[10] L.A. Lacerda, L.C. Wrobel, W.J. Mansur, A dual boundary element formulation for sound propagation around barriers over an infinite plane, Journal of Sound and Vibration 202 (1997) 235-347.

[11] L.A. Lacerda, L.C. Wrobel, H. Power, W.J. Mansur, A novel boundary integral formulation for three-dimensional analysis of thin acoustic barriers over an impedance plane, Journal of the Acoustical Society of America 104 (1998) 671-678.

[12] P. Jean, J. Defrance, Y. Gabillet, The importance of source type on the assessment of noise barriers, Journal of Sound and Vibration 226 (1999) 201-216.

[13] L. Godinho, J. António, A. Tadeu, 3D Sound scattering by rigid barrier in the vicinity of tall buildings, Applied Acoustics 62 (2001) $1229-1248$.

[14] L. Godinho, J. António, A. Tadeu, Sound propagation around rigid barriers laterally confined by tall buildings, Applied Acoustics 63 (2002) 595-609.

[15] F. Branco, L. Godinho, A. Tadeu, Acoustic insertion loss provided by rigid acoustic barriers of different shapes, Journal of Computational Acoustics 11 (2003) 503-519.

[16] A. Tadeu, L. Godinho, Scattering of acoustic waves by movable lightweight elastic screens, Engineering Analysis with Boundary Elements 27 (2003) 215-226.

[17] T. Pointer, E. Liu, J.A. Hudson, Numerical modeling of seismic waves scattered by hydrofractures: application of the indirect boundary element method, Geophysical Journal International 135 (1998) 289-303.

[18] T.A. Cruse, Boundary Element Analysis in Computational Fracture Mechanics, Kluwer Academic Publishers, Dordrecht, 1987.

[19] V. Sládek, J. Sládek, Transient elastodynamics three-dimensional problems in cracked bodies, Applied Mathematical Modelling 8 (1984) 2-10.

[20] V. Sládek, J. Sládek, A boundary integral equation method for dynamic crack problems, Engineering Fracture Mechanics 27 (1987) 269-277.

[21] K. Takakuda, Diffraction of plane harmonic waves by cracks, Bulletin of the JSME 214 (1983) 487-493.

[22] D. Prosper, Modeling and Detection of Delaminations in Laminated Plates, PhD Thesis, MIT Press, Cambridge, 2001.

[23] D. Prosper, E. Kausel, Wave scattering by cracks in laminated media, in: S.N. Atluri, T. Nishioka, M. Kikuchi (Eds.), Proceedings of the International Conference on Computational Engineering and Science ICES'01, Puerto Vallarta, 19-25/08/2001, Tech Science Press.

[24] M. Guiggiani, Formulation and Numerical Treatment of Boundary Integral Equations With Hypersingular Kernels. Singular Integrals in Boundary Element Methods, Computational Mechanics Publications, Southampton (UK) \& Boston (USA), 1998.

[25] E. Kausel, Forced vibrations of circular foundations in layered media. Department of Civil Engineering, MIT Research Report 70-3, MIT Press, Cambridge, USA, 1974.

[26] E. Kausel, J.M. Roesset, Frequency domain analysis of undamped systems, Journal of Engineering Mechanics ASCE 118 (1992) $721-734$

[27] C.L. Morfey, Dictionary of Acoustics, Academic Press, New York, 2001. 